\title{
Colorectal cancer patients with liver metastases and severe hyperbilirubinemia: A consecutive series that explores the benefits and risks of chemotherapy
}

\author{
Tamana Walia \\ J Fernando Quevedo \\ Timothy J Hobday \\ Gary Croghan \\ Aminah Jatoi \\ Division of Medical Oncology, \\ Rochester, MN, USA
}

Correspondence:Aminah Jatoi

200 First Street SW, Rochester, Minnesota 55905, USA

Email jatoi.aminah@mayo.edu
Background: Do colorectal cancer patients with hyperbilirubinemia and liver metastases benefit from chemotherapy?

Methods/Results: This study entailed a review of 3,019 consecutive patients with colorectal cancer. Within this cohort, 20 met the study's a priori selection criteria, which included a new diagnosis of colorectal cancer, no prior therapy, and a total bilirubin of $\geq 3.0 \mathrm{mg} / \mathrm{dL}$. All 20 patients had liver metastases, and as a whole the group had a median serum bilirubin of $6.4 \mathrm{mg} / \mathrm{dL}$ (range 3.1, $28 \mathrm{mg} / \mathrm{dL}$ ). Six patients received chemotherapy with an oxaliplatincontaining regimen, and four subsequently sustained a drop in their bilirubin. In one instance, a drop from 27.2 to $2.5 \mathrm{mg} / \mathrm{dL}$ occurred. These six patients lived a median of 71 days (range $23+, 283$ days), but one treatment-related death occurred. In contrast, patients who received only supportive care lived a median of 28 days.

Conclusion: Chemotherapy appears to provide modest benefit to newly diagnosed colorectal cancer patients with severe hyperbilirubinemia.

Keywords: colorectal cancer, liver metastases, hyperbilirubinemia, chemotherapy, oxaliplatin

Colorectal cancer is no longer considered a chemotherapy-resistant malignancy. A 1985 study outlined the benefits of first-line, single agent 5-fluorouracil, describing tumor response rates of only $23 \%$ and a median survival of only 8.7 months (Burocker et al 1985). No extra benefits were reported with the addition of other chemotherapy drugs (Burocker et al 1985).

Contrast these dismal results to more recent findings. Goldberg and colleagues (2004) demonstrated that a multidrug, first-line regimen, which consisted of oxaliplatin, leucovorin, and 5-fluorouracil, yielded a tumor response rate of $45 \%$, nearly double that reported earlier, and stretched the median survival by over two-fold, to 19.5 months. Such findings underscore the major therapeutic strides that have occurred in the management of patients with metastatic colorectal cancer and demonstrate that this previously chemotherapy-resistant malignancy can now be viewed as chemosensitive.

Have such breakthroughs changed our approach? Are we now at the point where patients, who were formerly considered marginal candidates for chemotherapy, can be prescribed it routinely? Specifically, should patients with liver metastases and marked hyperbilirubinemia now be prescribed chemotherapy in the hope of improving their hepatic function and improving their clinical outcomes? To our knowledge, these questions have not yet been answered because large, prospective clinical trials typically require that patients enroll with normal or near-normal organ function. Moreover, to date, only small case reports, some of which are not totally relevant to the questions 
posed above, have served to provide guidance on clinical management (Van Laethem et al 2003; Gupta et al 2007; Moosmann et al 2007). Hence, the purpose of the present study was to report outcomes within a group of colorectal cancer patients with severe hyperbilirubinemia presumably from liver metastases in an effort to provide more substantive guidance in answering the question of whether these patients benefit from state-of-the-art chemotherapy.

\section{Methods}

\section{Overview}

The Mayo Clinic Institutional Review Board approved the study protocol prior to its implementation. The Mayo Clinic Tumor Registry then provided information on all patients seen at the Mayo Clinic in Rochester, Minnesota USA with a diagnosis of colorectal cancer between the years of 2002 through 2006. The rationale for choosing 2002 as the start year rests in the fact that, by this time, oxaliplatin, an effective, non-liver metabolized drug, had been approved by the Food and Drug Administration for clinical use in colorectal cancer patients (Baldwin 2002).

\section{Screening process of medical records}

All medical records provided by the Tumor Registry were then screened by one investigator (TW) to identify patients who met the study team's a priori selection criteria. These criteria included severe hyperbilirubinemia (defined below), unresectable metastatic colorectal cancer, and no prior colorectal cancer therapy (no prior surgery, radiation, or chemotherapy). With regard to the latter point, a deliberate decision was made to select colorectal cancer patients with a newly diagnosed malignancy, as those with recurrent disease were likely to tolerate chemotherapy poorly as a result of prior surgery, radiation, or chemotherapy, thereby potentially biasing conclusions negatively (Ho et al 1996; Petersen et al 2007; Schneider et al 2007).

Severe organ dysfunction was initially defined on the basis of hyperbilirubinemia because of the proclivity of colorectal cancer to metastasize to the liver. Patients who had a serum total bilirubin of $3 \mathrm{mg} / \mathrm{dL}$ or greater in the absence of previous cancer therapy were the focus of this study. This threshold was chosen because another effective drug in colorectal cancer, irinotecan, is contraindicated at this serum bilirubin level for safety reasons (Schaaf et al 2006), a fact that emphasizes the grave nature of this degree of hyperbilirubinemia. Thus, it appeared reasonable to target patients with this degree of organ dysfunction and to utilize this serum bilirubin threshold in the screening process.

\section{In depth review of medical records}

The medical records of patients identified in this screening process were then reviewed in depth. One investigator (TW) reviewed these medical records, and another investigator (AJ) confirmed specific information.

Information on patient age at cancer diagnosis, gender, relevant laboratory data, method of assessing the presence of liver metastases, whether liver metastases were in fact present, the number and type of chemotherapy regimens prescribed, whether a biliary stent was placed, adverse events, and overall survival were gleaned from each medical record. Tumor response was not assessed because of inconsistent patterns in radiographic testing from patient to patient and because of the greater value placed on survival (ASCO 1996; Therasse et al 2000). It was also decided not to attempt to glean information on performance status in view of inconsistent reporting and attribution from physician to physician (Sorensen et al 1993).

\section{Data analyses}

This study was intended to be descriptive; all demographic and outcome data are presented as such. Survival is defined as the interval between cancer diagnosis and date of death. In the event that the date of death was unknown, data were censored from the date of last follow up. JMP version 6 (SAS Inc., Cary, NC) was used to construct Kaplan-Meier survival curves.

\section{Results}

\section{Patient demographics}

A total of 3,019 patients were initially identified by the Mayo Clinic Tumor Registry. The vast majority was found to have a serum bilirubin $<3 \mathrm{mg} / \mathrm{dL}$ and/or to have received prior cancer treatment. Nonetheless, the screening process yielded 20 patients who met the selection criteria.

Among these 20 patients, 11 were male (55\%), and the median age at colorectal cancer diagnosis was 54 years (range 39, 81) (Table 1). Only 2 had rectal cancer, and the remaining colon cancer. The median bilirubin level was $6.4 \mathrm{mg} / \mathrm{dL}$ (range 3.1, $28 \mathrm{mg} / \mathrm{dL}$ ). All had liver metastases, as seen by computed tomography (17 patients), ultrasound (1 patient), or surgical exploration or biopsy (2 patients). Hyperbilirubinemia was not attributable to hemolysis in any patient, and, in effect, review of the medical record did not show any apparent nonbiliary or nonhepatic cause.

Six patients received chemotherapy, and 12 received only supportive care. In two patients, the medical record did 
Table I Baseline characteristics $(n=20)$

\begin{tabular}{ll}
\hline Age (in years) & \\
Median (range) & $54(39,8 \mathrm{I})$ \\
Gender & $\mathrm{II}(55)$ \\
Male (\%) & $9(45)$ \\
Female (\%) & \\
Serum bilirubin (in $\mathrm{mg} / \mathrm{dL})$ & $6.4(3.1,28)$ \\
Median (range) & \\
Cancer site & $18(90)$ \\
Colon (\%) & $2(10)$ \\
\hline
\end{tabular}

not document chemotherapy administration or the decision to resort to supportive care exclusively, but one of these patients was alive at 394 days after diagnosis. In this patient, internal and external records show a recommendation to proceed with an oxaliplatin-based regimen in combination with bevacizumab. Well documented, first-line chemotherapy in the other patients included: oxaliplatin, 5-fluorouracil, and leucovorin (three patients); oxaliplatin, 5-fluorouracil, leucovorin, and bevacizumab (one patient); and oxaliplatin and capecitabine (one patient). Second-line chemotherapy that consisted of irinotecan, 5-fluorouracil, and leucovorin was administered to one patient.

The median survival of the six chemotherapy-treated patients was 71 days. Among the 12 nontreated patients, it was 28 days (Figure 1).

Chemotherapy and/or biliary stent placement was associated with a drop in bilirubin in four patients (Table 2). Chemotherapy alone was associated with a decrease in bilirubin in two patients, leading to a decline from 27.7 to $2.5 \mathrm{mg} / \mathrm{dL}$ in one and a modest decline from 6.5 to $5.7 \mathrm{mg} / \mathrm{dL}$ in another. It is impossible to say that this decline is definitely attributable to the chemotherapy. In five patients, a stent was placed, and two of these patients also received chemotherapy. It appears that the nonstented patients had hepatic dysfunction related to intra-parenchymal liver disease. In one of these stented, chemotherapy-treated patients, the bilirubin dropped from $16.2 \mathrm{mg} / \mathrm{dL}$ to a normal level of $0.6 \mathrm{mg} / \mathrm{dL}$; and, in the other, it dropped from $12.5 \mathrm{mg} / \mathrm{dL}$ to $3 \mathrm{mg} / \mathrm{dL}$. In the three other patients who underwent only stent placement, the bilirubin stayed the same in one, increased in another, and apparently was not rechecked in the third.

In terms of adverse events, severe fatigue was noted in one patient who received chemotherapy. Another suffered from mucositis and dehydration after chemotherapy.

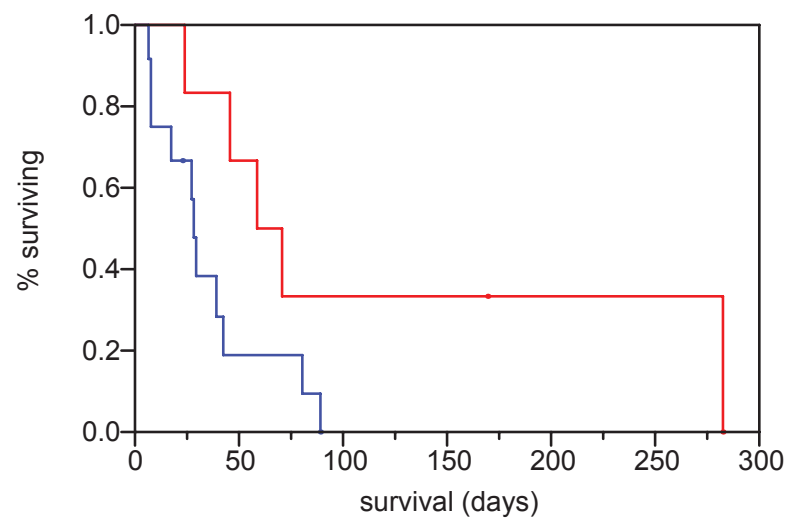

Figure I Median survival among the chemotherapy-treated patients was 7 I days (red line), and among nontreated patients 28 days (blue line).

This same patient was hospitalized and died from a gastrointestinal bleed approximately five weeks after receiving bevacizumab (Patient \#2 in Table 2).

\section{Discussion}

To our knowledge, this study is one of the few to evaluate outcomes in colorectal cancer patients with liver metastases and severe hyperbilirubinemia. Overall, these 20 patients did poorly. None achieved the median survival of 19.5 months, as reported by Goldberg and colleagues (2004); and, in fact, only one was confirmed to live beyond the one-year mark. The observations that patients who received chemotherapy lived relatively longer and at times manifested a decline in bilirubin suggest that chemotherapy was providing antineoplastic effects. Hence, a small subgroup of patients with colorectal cancer, liver metastases, and severe hyperbilirubinemia appears to derive modest benefits from chemotherapy.

It is important to point out the limitations of this study. First, patients who received chemotherapy may have been more fit, better able to tolerate it, and therefore more likely to derive benefit from it. In the absence of a randomized, controlled trial, the best we can conclude is that a subgroup of patients with hyperbilirubinemia appears to benefit. It remains unclear whether chemotherapy provides better outcomes compared to supportive care alone. Second, although this study observed that one patient did die from a chemotherapy-related complication, the retrospective design of this study might predispose to the underreporting of adverse events. Thus, we cannot claim that the risk to benefit ratio of chemotherapy clearly justifies its administration, despite the fact that the median survival was greater among chemotherapy-treated patients. Third, although it is tantalizing to conclude that perhaps patients with hyperbilirubinemia in the setting of other malignancies would also potentially benefit from 
Table 2 Outcomes among the nine chemotherapy-treated and/or stented patients

\begin{tabular}{|c|c|c|c|c|c|c|c|c|c|}
\hline & $\mathbf{I}$ & 2 & 3 & 4 & 5 & 6 & 7 & 8 & 9 \\
\hline Age (years) & 56 & 81 & 80 & 49 & 51 & 46 & 62 & 53 & 57 \\
\hline Gender & Male & Female & Male & Male & Male & Male & Male & Male & Male \\
\hline $\begin{array}{l}\text { Total bilirubin at } \\
\text { diagnosis (mg/dL) }\end{array}$ & 16.2 & 27.7 & 13.8 & 6.2 & 4.4 & 3.5 & 12.5 & 6.5 & 3.2 \\
\hline $\begin{array}{l}\text { Stent placement } \\
\text { at diagnosis }\end{array}$ & Yes & No & Yes & No & Yes & No & Yes & No & Yes \\
\hline Chemotherapy & $\begin{array}{l}\text { Oxaliplatin, } \\
\text { 5-fluorouracil, } \\
\text { Leucovorin, } \\
\text { then irinotecan, } \\
\text { 5-fluorouracil, } \\
\text { leucovorin }\end{array}$ & $\begin{array}{l}\text { Oxaliplatin, } \\
\text { 5-fluorouracil, } \\
\text { Leucovorin, } \\
\text { bevacizumab }\end{array}$ & None & $\begin{array}{l}\text { Oxaliplatin, } \\
\text { capecitabine }\end{array}$ & None & $\begin{array}{l}\text { Oxaliplatin, } \\
\text { 5-fluorouracil, } \\
\text { Leucovorin, }\end{array}$ & $\begin{array}{l}\text { Oxaliplatin, } \\
\text { 5-fluorouracil, } \\
\text { Leucovorin, }\end{array}$ & $\begin{array}{l}\text { Oxaliplatin, } \\
\text { 5-fluorouracil, } \\
\text { Leucovorin, } \\
\text { bevacizumab }\end{array}$ & Unknown \\
\hline Drop in bilirubin? & Yes & Yes & No & No & No & No & Yes & Yes & Unknown \\
\hline Survival (days) ${ }^{*}$ & 283 & 59 & 86 & 24 & $23+$ & 46 & $170+$ & 71 & $394+$ \\
\hline
\end{tabular}

Note: *Censored data are denoted by a + sign.

chemotherapy, this conclusion would be perilous. Further study of hyperbilirubinemia in other cancer settings is indicated before such conclusions can be drawn.

The question of whether or not to attempt to place a stent in patients with biliary obstruction also remains unanswered. This study included only two patients who had had a biliary stent placed and who did not receive subsequent chemotherapy. These two patients manifested little clinical improvement. Although no firm conclusions can be drawn, we might begin to question the value of stenting a patient in the absence of plans to initiate subsequent chemotherapy. This issue perhaps merits further study.

In summary, the present study provides justification for prescribing chemotherapy to patients with colorectal cancer, liver metastases, and hyperbilirubinemia. However, given the poor outcome in this series as a whole, it remains prudent to continue to rely on clinical judgment in making therapeutic decisions and to discuss risks and benefits with patients and their families at length prior to prescribing chemotherapy.

\section{Disclosure}

The authors report no conflicts of interest in this work.

\section{References}

[ASCO] American Society of Clinical Oncology. 1996. Outcomes of cancer treatment for technology assessment and cancer treatment guidelines. American Society of Clinical Oncology. J Clin Oncol, 14:671-9.

Baldwin J. 2002. FDA evaluating oxaliplatin for advanced colorectal cancer. J Natl Cancer Inst, 94:1191-3.
Burocker TR, Moertel CG, Fleming TR, et al. 1985. A controlled evaluation of recent approaches to biochemical modulation or enhancement of 5-fluorouracil therapy in colorectal cancer. J Clin Oncol, 3:1624-31.

Goldberg RM, Sargent DJ, Morton RF, et al. 2004. A randomized controlled trial of fluouracil plus leucovorin, irinotecan, and oxaliplatin combinations in patients with previously untreated metastatic colorectal cancer. J Clin Oncol, 22:23-30.

Gupta A, LeVea C, Litwin A, et al. 2007. Reversible grade 4 hyperbilirubinemia in a patient with UGT1A1 7/7 genotype treated with irinotecan and cetuximab. Clin Colorectal Cancer, 6:447-9.

Ho YH, Low D, Goh HS. 1996. Bowel function survey after segmental colorectal resections. Dis Colon Rectum, 39:307-10.

Moosmann N, Laessig D, Michaely HJ, et al. 2007. Effective second-line treatment with cetuximab and bevacizumab in a patient with hepatic metastases of colorectal cancer and hyperbilirubinemia. Onkologie, 30:509-12.

Petersen S, Jongen J, Petersen C, et al. 2007. Radiation-induced sequelae affecting the continence organ: incidence, pathogenesis, and treatment. Dis Colon Rectum, 50:1466-74.

Schaaf LJ, Hammond LA, Tipping SJ, et al. 2006. Phase I and pharmacokinetic study of intravenous irinotecan in refractory solid tumor patients with hepatic dysfunction. Clin Cancer Res, 12:3782-91.

Schneider EC, Malin JL, Kahn KL, et al. 2007. Surviving colorectal cancer: patient-reported symptoms 4 years after diagnosis. Cancer, 110:2075-82.

Sorensen JB, Klee M, Palshof T, et al. 1993. Performance status assessment in cancer patients. An inter-observer variability study. Br J Cancer, 67:773-5.

Therasse P, Arbuck SG, Eisenhauer EA, et al. 2000. New guidelines to evaluate the response to treatment of solid tumors. European Organization for Research and Treatment of Cancer, National Cancer Institute of the United States, National Cancer Institute of Canada. J Natl Cancer Inst, 92:205-16.

Van Laethem JL, De Broux S, Eisendrath P, et al. 2003. Clinical impact of biliary drainage and jaundice resolution in patients with obstructive metastases at the hilum. Am J Gastroentrol, 98:1221-2. 